\title{
Mutation Analysis of BRCA1 and BRCA2 in Italian Hereditary and Sporadic Forms of Breast and Ovarian Cancers: Tumor Genotype-Phenotype Correlation in Breast Cancer BRCA-Mutation Carriers
}

\author{
G. Cipollini, C. Ghimenti, E. Sensi, \\ D. Iandolo, A. Piccirilli, A. Berti, \\ G. Naccarato, P. Viacava, D. Campani, \\ G. Bevilacqua and M.A. Caligo \\ Department of Oncology, Section of \\ Pathology, University of Pisa, Italy
}

Predisposition to breast and ovarian cancer has been attributed to mutant BRCA1 alleles in $90 \%$ of hereditary combined tumors and in $45 \%$ of hereditary breast cases, whereas mutations in BRCA2 gene are thought to account for about $35 \%$ of inherited breast cancers. On the other hand, the presence of mutations in the sporadic forms of these tumors is an infrequent event. This suggests that tumors arising in BRCA mutation carriers may differ from BRCA negative hereditary and sporadic cancer in genetic and biological features as well as in clinical behaviour.

We undertook our study to evaluate the frequent of BRCA mutations in patients with hereditary and sporadic breast and/or ovarian tumors in order to explore if specific pathological and clinical features of tumors are associated with BRCA1 and BRCA2 mutations.

Mutational analysis was also performed for exon 11 by PTT complemented by SSCP for the remaining entire coding region and exon-intron splice boundaries. Direct sequence analysis was used to identify the genetic alterations.

DNA germline mutations were detected in 22 out 76 (29\%) families; 15 probands (20\%) showed BRCA1 mutations; 7 patients (9\%) presented mutations in the BRCA2 gene. About $50 \%(11 / 22)$ BRCA-mutations were detected in families with three or more affected relatives most of which (8/11) occurred in BRCA1;27\% (6/22) BRCA-mutations were observed in families with two affected relatives; $18 \%$ (4/22) in families with one affected relative and one BRCA-mutation $(5 \% ; 1 / 22)$ was identified in a borderline family. No hot spot of mutations was found in the two genes, but almost 50\% occurred in exon 11 of BRCA1 and BRCA2.

The BRCA-mutational analysis, performed on 30 primary early onset breast cancers $(\leq 36$ yrs.) and 18 ovarian carcinomas ( $\leq 50 \mathrm{yrs}$.), revealed the presence of: a) one somatic point mutation, an $5382 \mathrm{insC}$, in one ovarian cancer patient, b) a rare sequence variant in exon 15 in one case of breast cancer, c) four different common polymorphisms (one in intron 8 in breast and ovarian tumors, one in intron 7 in breast carcinomas and one in exon 13 and in exon 16 in ovarian neoplasm).

The specific pathological and clinical features of breast-tumors associated with BRCA1 and BRCA2 mutations were explored (histological type, pathological grade, lymph node status, tumor size, lymphatic and blood vessel tumor emboli, tumor necrosis, presence of other lesions in the parenchyma collateral to invasive car- 
cinoma, estrogen-progesterone receptors). Three groups of breast tumors were compared: BRCApositive hereditary-breast cancer, BRCAnegative hereditary-breast cancers and sporadic early onset breast cancers.

Data from this study showed that: a) the tumors isolated from women carriers of BRCA1 and BRCA2 mutations were more often associated with negativity for estrogen receptors and with presence of lymph node metastasis; b) BRCA positive tumors were of higher nuclear grade; c) familial breast-cancer tumors, independently of BRCA1 and BRCA2 mutational status, showed a statistically significant association with lymphatic vessel infiltration and necrosis.

Our results show that BRCA mutations are present in $29 \%$ of familial breast-ovarian cancers, in $5 \%$ of sporadic ovarian tumors and that no somatic alteration occurs in sporadic breast cancer. It is possible that sporadic and hereditary breast-ovarian cancers have different mutational spectra. Moreover, we confirmed that the BRCA1 and BRCA2 associated breast carcinomas present worse histopathological features than sporadic ones, matched by age of diagnosis. 


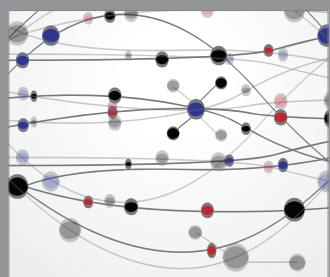

The Scientific World Journal
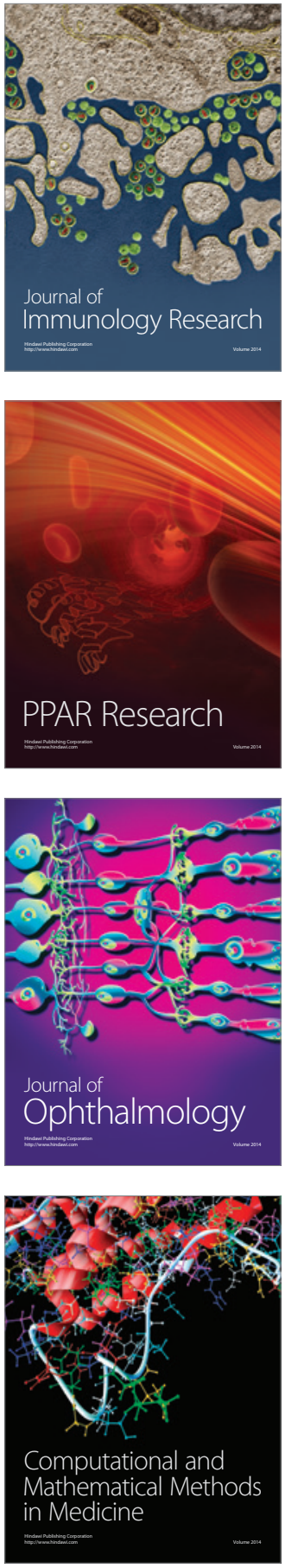

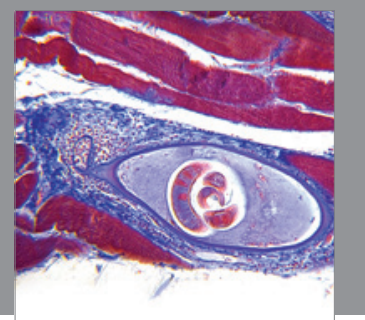

Gastroenterology

Research and Practice
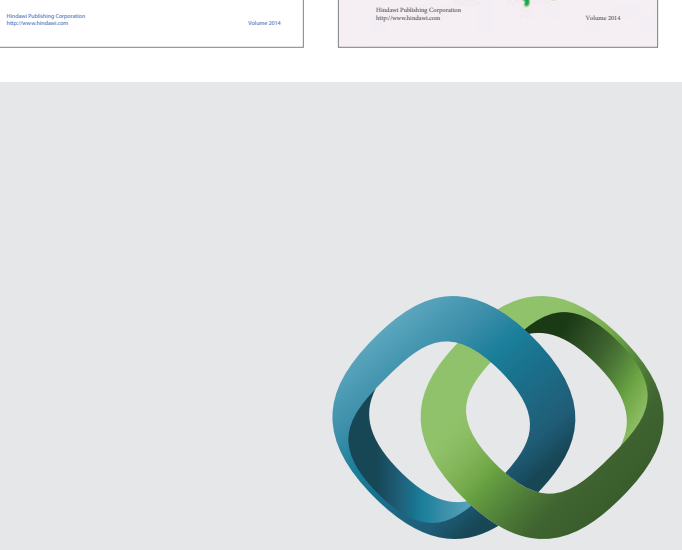

\section{Hindawi}

Submit your manuscripts at

http://www.hindawi.com
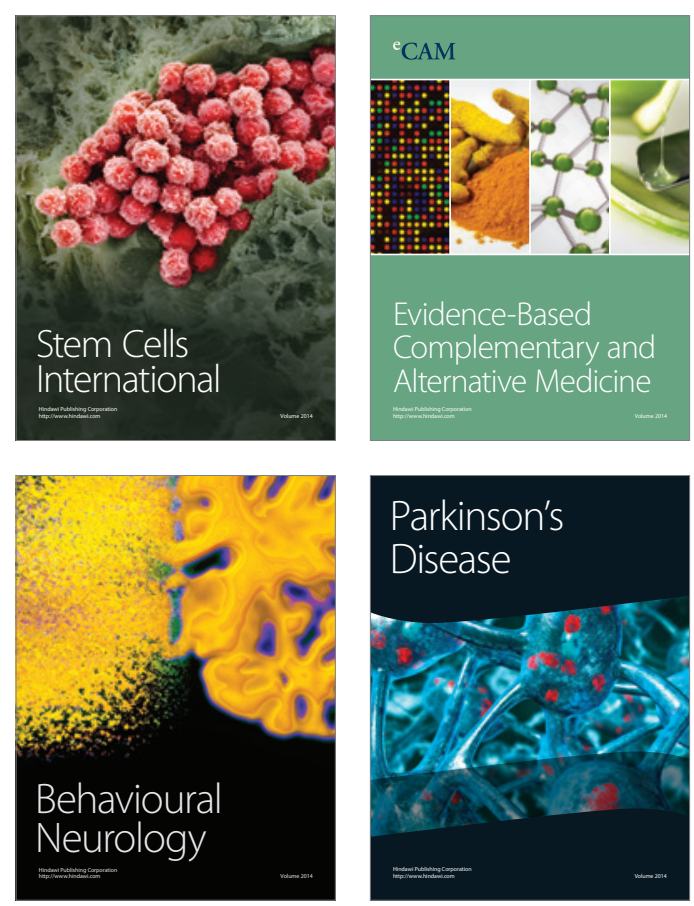

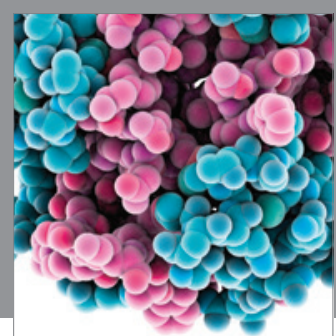

Journal of
Diabetes Research

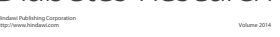

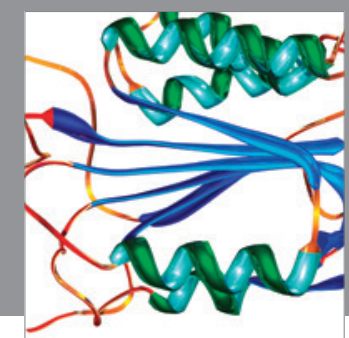

Disease Markers
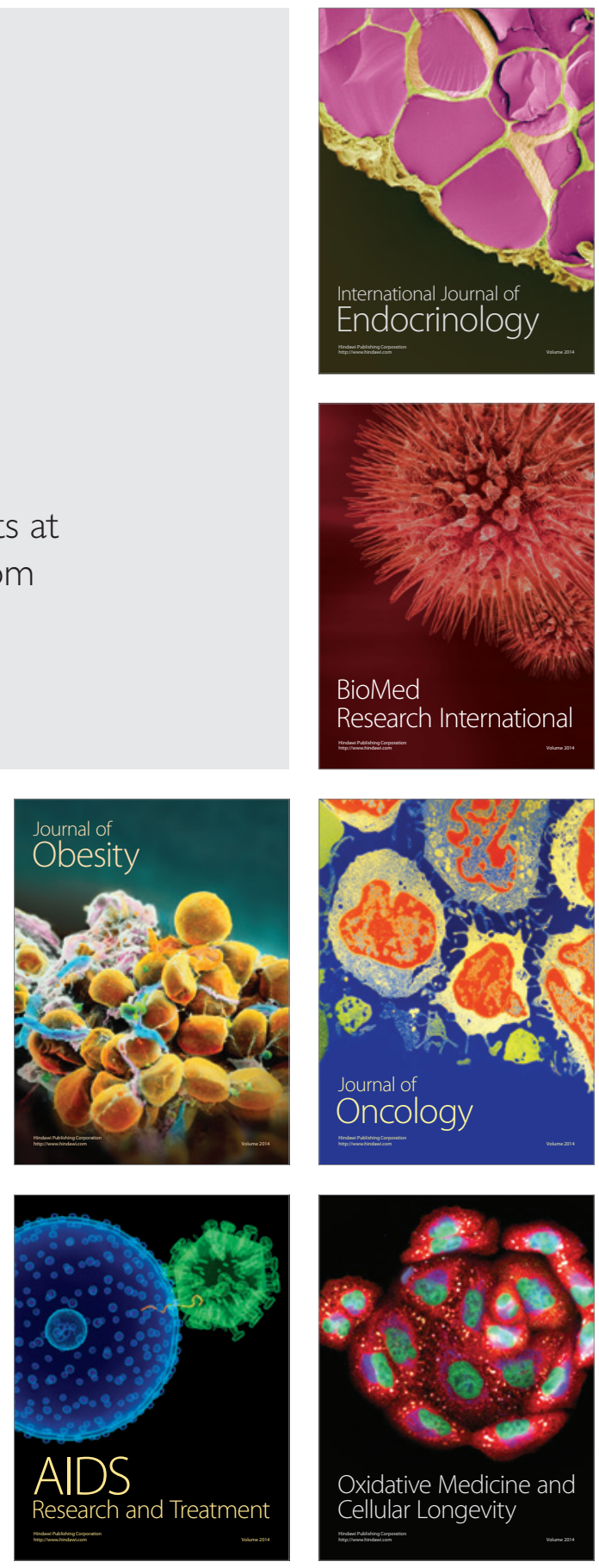